\title{
Producción de semillas de Araucaria araucana (Molina) K. Koch durante 15 años en diferentes poblaciones del Parque Nacional Lanín (Neuquén-Argentina)
}

\author{
JAVIER SANGUINETTI ${ }^{\bowtie}$ \\ Parque Nacional Lanín, Administración de Parques Nacionales, San Martín de los Andes, Argentina
}

\begin{abstract}
REsumEn. Los estudios sobre producción de semillas en especies arbóreas son importantes para la ciencia y para el manejo de los bosques nativos. En este trabajo se estudió la producción a largo plazo de conos de araucaria (Araucaria araucana), cuyas semillas son utilizadas como alimento por el ser humano, en poblaciones ubicadas en distinta exposición y altitud, y con diferente composición y estructura forestal. Se contaron conos en siete poblaciones del Parque Nacional Lanín (Neuquén, Argentina) durante 15 años (2000 a 2014), y se estimó la cantidad de conos y semillas por árbol, por hectárea y por población. Los años de máxima producción fueron 2000, 2007 y 2013, mientras que los de mínima fueron 2005, 2008, 2012 y 2014. La producción media estimada de conos por árbol fue 19 (rango: 1-62); mientras que la de semillas fue 2372 (rango: 61-9287). La mayor variación temporal en la producción de semillas respecto a la de conos se debió a que hubo significativamente más semillas por cono en los años más productivos. El promedio estimado por hectárea fue de 685 conos, 89394 semillas y 273 kilos de semilla. Los bosques puros y densos de araucaria y los mixtos con lenga son, en promedio, al menos 2.5 y 1.5 veces más productivos que el resto de los bosques. El número de conos por árbol varío entre poblaciones y asociado principalmente con la exposición solar. Sin embargo, este efecto difiere según la productividad anual y el patrón observado estaría vinculado con el mecanismo de formación de yemas florales estimulado por la sequía. Estos resultados pueden utilizarse para planificar la conservación de estos bosques en Argentina y Chile frente a la mayor presión en la cosecha de semillas y por la presencia de granívoros introducidos.
\end{abstract}

[Palabras clave: conos, exposición solar, reproducción]

\begin{abstract}
Aвstract. Araucaria araucana (Molina) K. Koch seed production during 15 years at different populations in Lanín National Park (Neuquén-Argentina): Seed production studies on tree species are important for science and for forest management. The objective of this work was to study the long term seed production of araucaria (Araucaria araucana), which are a valuable food for humans, at populations located at different aspect, altitude and with variations on forest structure and composition. Cones were counted at seven populations in Lanín National Park, Neuquén Province, Argentina for 15 years (2000 to 2014), estimating seeds and cones per tree, per hectare and per population. Peaks in seed production occurred in 2000, 2007 and 2013, while seed crops were low during 2005, 2008, 2012 and 2014. Mean cones per tree was 19 (range: 1-62) with 56 fold variation among years. Mean seeds per tree was 2372 (range: 61-9287) with 152 fold variation among years. The greater temporal variation in seed production in relation to cones was due to a significant increase in seeds per cone during mast years. Mean cones, individual seeds and kilograms of seeds per hectare were 685, 89394 and 273, respectively. Dense and pure Araucaria forests and those mixed with lenga (Nothofagus pumilio) were at least 2.5 and 1.5 fold more productive than the rest of forest types. Cones per tree differed significantly among sites and were related mainly with sun exposure. However, this effect varied according to the class productive year and the observed pattern may be related with the floral bud formation associated with drought. Araucaria showed a synchronous reproduction between trees and populations at regional scale, with a greater temporal variability than spatial variation between populations. These results could be important for conservation planning of these forests in Argentina and Chile against the threats posed by increased seed gathering pressure and by the presence of exotic invasive granivores.
\end{abstract}

[Keywords: cones, sun exposure, reproduction]

\section{INTRODUCCIÓN}

Los estudios sobre producción de semillas en árboles son de gran interés tanto desde el punto de vista científico como desde el de la conservación y el uso sustentable (Harper 1977). Conocer el patrón temporal y la magnitud de la producción de semillas puede ayudar a los científicos a entender el proceso

Editora asociada: Ana Cingolani

$\square$ sanguinetti.javier@gmail.com ecológico representado por la producción y dispersión de semillas y su vinculación con los factores que limitan la reproducción y la regeneración (Herrera et al. 1998). También este conocimiento puede ayudar a los administradores y usuarios de estas especies a definir pautas de manejo, especialmente cuando son un recurso natural utilizado por el humano (Lara et al. 1995). 
La producción de semillas en árboles es un proceso complejo y en el caso de las especies de coníferas puede durar dos o tres años (Owens \& Blake 1985). Las etapas de este proceso incluyen el desarrollo y diferenciación (inducción floral) de yemas para la formación de estróbilos, la formación de óvulos y su posterior fecundación vía polinización generalmente por viento y finalmente, el desarrollo y crecimiento de las semillas y de los conos que las protegen (Owens \& Blake 1985). Cada una de estas etapas está controlada por numerosos factores endógenos y exógenos que interactúan entre sí; en condiciones naturales es casi imposible separar la contribución de cada uno y estudiar todas las interacciones entre ellos (Owens \& Blake 1985).

A escala regional, las diferencias entre sitios en la productividad de semillas puede deberse a variaciones en la disponibilidad de recursos (e.g., fósforo o nitrógeno), ya sea por efecto del clima, de la topografía (exposición y/o altitud) o por diferencias en la fertilidad de los suelos (Mathews 1963; Worrell \& Malcom 1989). A escala local, los árboles pueden presentar diferencias en su productividad reproductiva debido al efecto de la competencia intra o interespecífica frente a nutrientes o luz limitantes (Mattews 1963; Greene et al. 2002; Meunier et al. 2007).

La araucaria (Araucaria araucana) es una conífera presente en el norte de Patagonia en un rango altitudinal amplio (1000-1800 m.s.n.m.) y sobre distintas exposiciones y condiciones de suelo, formando comunidades boscosas puras o mixtas con especies del género Nothofagus spp. (Veblen et al. 1995). En este contexto, es de esperar que existan diferencias en la producción de conos entre poblaciones sometidas a diferentes condiciones abióticas y bióticas.

Las semillas ("piñones") de araucaria constituyen un recurso valioso para humano (para el Pueblo Mapuche o "Pehuenche") desde tiempos ancestrales (Herrmann 2006). Sin embargo, las presiones actuales del mercado y las demandas futuras por este recurso desde los centros urbanos pueden generar escenarios negativos de conservación, con incrementos significativos en los niveles de recolección en comparación con la productividad de los bosques.

La araucaria tiene elevada prioridad de conservación a nivel nacional e internacional por su valores ecológicos, estéticos, paisajísticos y espirituales (Veblen et al. 1995). Enfrenta amenazas diversas como la ganadería, la recolección excesiva de semillas y de leña por el ser humano, la invasión de pinos exóticos plantados masivamente y de mamíferos invasores, o la ocurrencia de incendios severos (Rechene et al. 2003; Shepherd \& Ditgen 2005; Sanguinetti 2008; Sanguinetti \& Kitzberger 2010; Zamorano-Elgueta et al. 2012). En este contexto, y considerando su fecundidad reducida, su baja capacidad de dispersión y, por ende, su limitado nivel de regeneración (Veblen 1982), se considera clave generar información sobre la magnitud de la producción de semillas en esta especie, conocer su variabilidad espacial y temporal y los factores que la influyen.

Escasos trabajos estiman la producción de semillas de araucaria por hectárea y por bosques, y casi todos son de corto plazo (Muñoz Ibáñez 1984; Caro 1995; Donoso et al. 2010). Sanguinetti \& Kitzberger (2008) determinaron entre 2000-2008 que la especie presenta una producción de conos por árbol altamente sincronizada a nivel regional entre individuos y entre poblaciones. El presente trabajo busca profundizar los estudios previos al estimar la producción de conos y semillas por árbol, por hectárea y por población (subcuenca), y al evaluar las diferencias de productividad en bosques ubicados a distinta altitud y exposición, lo cual determina que presenten diferencias en composición y estructura. Los objetivos del trabajo son: a) aportar información de largo plazo sobre la producción de semillas de araucaria a distintas escalas espaciales, b) comparar la productividad de conos entre poblaciones ubicadas a distinta altitud y exposición solar, y c) comparar la productividad de bosques con distinta composición y estructura.

\section{Materiales y MÉTOdos}

\section{Área de Estudio}

El estudio fue realizado en la Provincia del Neuquén, Argentina, entre los $39^{\circ} 12^{\prime} 37^{\prime \prime}$ y los

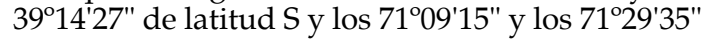
de longitud $\mathrm{O}$, en el norte del Parque Nacional Lanín (Figura 1). La precipitación en el área de estudio oscila entre 1500 y $2600 \mathrm{~mm}$ /año según el gradiente de humedad Este-Oeste. Las lluvias ocurren principalmente en el período frío (abril a septiembre). Los veranos suelen ser secos (De Fina 1972). En un área de $\sim 1540 \mathrm{~km}^{2}$, se seleccionaron siete poblaciones de araucaria, distanciadas entre 7 y $46 \mathrm{~km}$, para el estudio de la producción de conos durante 15 años (Tabla 1; Figura 1). Además, en 


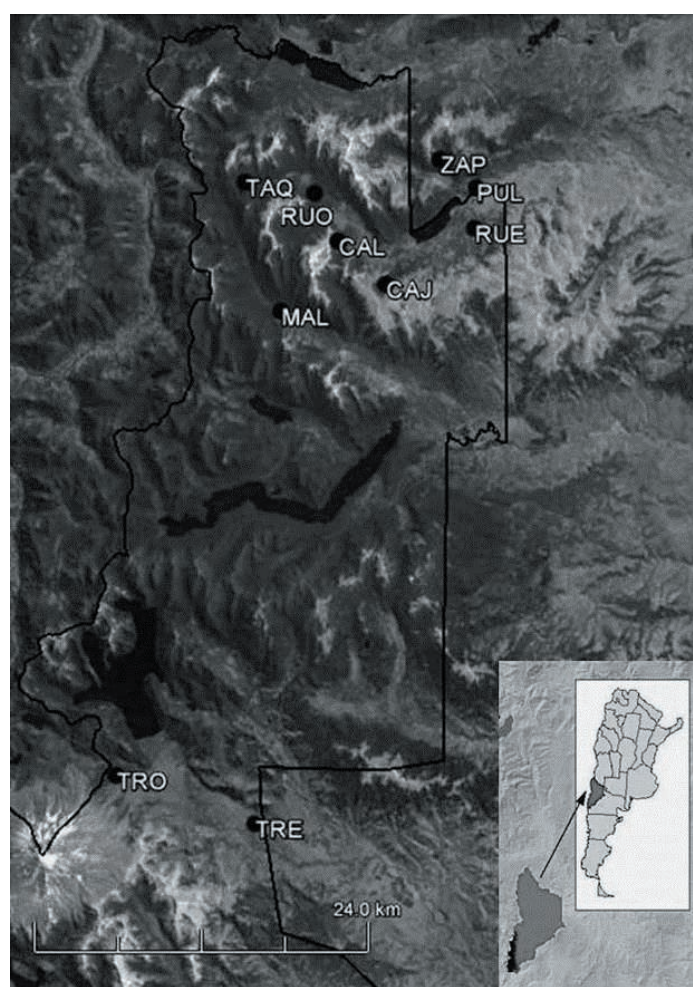

Figura 1. Mapa del SO de la Provincia del Neuquén, en e norte del Parque Nacional Lanín, mostrando la ubicación de las diez poblaciones de Araucaria araucana evaluadas y el límite del área protegida.

Figure 1. Map of southwestern Neuquén province, north of Lanín National Park, showing the location of the ten populations of Araucaria araucana evaluated and the boundary of the protected area.

otras tres poblaciones sólo se muestreó la densidad de semilleros y se estimó la productividad de conos por extrapolación utilizando los datos obtenidos en las otras siete poblaciones. Las poblaciones estudiadas presentan una superficie neta de araucaria que varía entre 50 y 500 ha.

La vegetación de la zona está caracterizada por la presencia de bosques de araucaria que forman distintas asociaciones con especies del género Nothofagus. Por lo general, los bosques mixtos de araucaria y lenga [N. pumilio (Poepp. \& Endl)] se ubican en las porciones más altas de las montañas, por encima de 1200 m.s.n.m., mientras que la asociación con ñire [N. antarctica (G. Forst)], en los valles y en las zonas bajas de los faldeos más secos con exposición norte. La araucaria forma bosques puros en sectores con actividad volcánica reciente o en suelos rocosos, pobres en materia orgánica (Rechene et al. 2003). En este trabajo, los bosques estudiados se clasificaron en: araucaria pura y densa (Aa_denso) o abierta (Aa_abierto) , araucaria dominante y acompañada por lenga (Aa $\mathrm{Np}$ ) o por ñire (Aa_Na), lenga o ñire dominantes y acompañada por araucaria (Np_Aa y Na_Aa, respectivamente) (Tabla 1 ).

\section{Especie estudiada}

Araucaria araucana (familia Araucariaceae) es una conífera endémica, emergente y longeva (>900 años) (Burns 1993) de los bosques templados de Chile y Argentina (Veblen et al. 1995). Tiene una distribución restringida entre los $37^{\circ} 20^{\prime}$ y los $40^{\circ} 20^{\prime} \mathrm{S}$, cubriendo 400000 ha en ambos países. La araucaria está adaptada a condiciones de estrés lumínico e hídrico, es tolerante a la sombra y crece principalmente en suelos menos productivos. Presenta adaptaciones al fuego tales como corteza gruesa y yemas epicórmicas que rebrotan luego de un incendio. Estas y otras adaptaciones permiten que araucaria coexista con las especies más competitivas del género Nothofagus spp.; los

Tabla 1. Ubicación y características generales de los sitios donde se estimó la producción de conos y la densidad de semilleros en el norte del Parque Nacional Lanín. Los datos sobre diámetro a la altura del pecho (Dap) y Área Basal (AB) son exclusivamente de A. araucana (Dap $>20 \mathrm{~cm}$ ). Los sitios Zapata, Pulmarí, Cahuel Cura, El Cajón, Calfiquitra, Lonco Pan y Taquinquin son de Rucachoroy y están presentados de Este a Oeste. En los sitios Zapata, Pulmarí, y El Cajón sólo se estimó la densidad de semilleros. Araucaria denso (Aa_denso), Araucaria-N. pumilio (Aa_Np), Araucaria-N. antarctica (Aa_Na), Araucaria abierto (Aa_abierto), N. pumilio-Araucaria (Np_Aa) y N. antartica-Araucaria (Na_Aa).

Table 1. Location and general characteristics of the sites where cone production and seeding tree densities were estimated. Dbh (Dap) and Basal Area (AB) are from A. araucana $(\mathrm{Dbh}>20 \mathrm{~cm})$. The sites Zapata, Pulmarí, Cahuel Cura, Calfiquitra, Lonco Pan y Taquinquin are located in Rucachoroy from East to West. At Zapata, Pulmarí and El Cajón sites only tree density was sampled. Dense Araucaria (Aa_denso), Araucaria-N. pumilio (Aa_Np), Araucaria-N. antarctica (Aa_Na), Open Araucaria (Aa_abierto), N. pumilio-Araucaria (Np_Aa) and N. antarctica-Araucaria (Na_Aa).

\begin{tabular}{lccccccc}
\hline Sitio & Sigla & Ubicación & $\begin{array}{c}\text { Altitud } \\
(\mathrm{msnm})\end{array}$ & Exposición & Tipo Bosque & $\begin{array}{c}\text { Dap } \\
(\mathrm{cm})\end{array}$ & $\begin{array}{c}\text { AB } \\
\left(\mathrm{m}^{2} / \mathrm{ha}\right)\end{array}$ \\
\hline Zapata & ZAP & $39^{\circ} 12^{\prime} / 71^{\circ} 10^{\prime}$ & 1350 & $\mathrm{~S}$ & Aa_abierto y denso, Aa_Np & 60 & 59.6 \\
Pulmarí & PUL & $39^{\circ} 13^{\prime} / 71^{\circ} 10^{\prime}$ & 1260 & E y sin exposición & Aa_Na y Aa_abierto & 51.6 & 23.8 \\
Cahuel Cura & RUE & $39^{\circ} 14^{\prime} / 71^{\circ} 10^{\prime}$ & 1360 & $\mathrm{~N}$ & Aa_abierto y denso, Aa_Na & 52.8 & 17.6 \\
El Cajón & CAJ & $39^{\circ} 15^{\prime} / 71^{\circ} 13^{\prime}$ & 1500 & $\mathrm{~N}$ & Aa_Np, Np_Aa & 54 & 45.8 \\
Calfiquitra & CAL & $39^{\circ} 14^{\prime} / 71^{\circ} 16^{\prime}$ & 1458 & $\mathrm{~N}$ y sin exposición & Aa_denso, Aa_Na & 42.3 & 16.5 \\
Lonco Pan & RUO & $39^{\circ} 13^{\prime} / 71^{\circ} 17^{\prime}$ & 1348 & Sin exposición & Aa_Na & 44.3 & 27.6 \\
Taquinquin & TAQ & $39^{\circ} 12^{\prime} / 71^{\circ} 21^{\prime}$ & 1550 & Sin exposición & Aa_Np & 78.3 & 34.9 \\
Malalco & MAL & $39^{\circ} 18^{\prime} / 71^{\circ} 17^{\prime}$ & 1160 & N & Aa_Na & 42.2 & 10.3 \\
Tromen oeste & TRO & $39^{\circ} 35^{\prime} / 71^{\circ} 27^{\prime}$ & 1210 & Sin exposición & Aa_Np,Aa_Na & 61.6 & 36 \\
Tromen este & TRE & $39^{\circ} 37^{\prime} / 71^{\circ} 21^{\prime}$ & 1000 & Sin exposición & Aa_Na,Aa_abierto & 47.2 & 19.8 \\
\hline
\end{tabular}


disturbios naturales juegan un papel relevante en esta interacción interespecífica (Veblen 1982).

La araucaria presenta una arquitectura de copa bastante particular, los individuos jóvenes suelen tener una forma cónica pero a medida que crecen van perdiendo las ramas inferiores para alcanzar la llamativa y típica forma de copa aparasolada. Esta transformación de la copa se acelera si los individuos crecen en condiciones de competencia y/o si lo hacen en sitios con suelos menos fértiles (Grosfeld 1994). Los árboles alcanzan la madurez sexual cuando presentan fustes mayores a $20 \mathrm{~cm}$ de diámetro y tienen más de 30 años de edad (Muñoz Ibáñez 1984). La araucaria es dioica (raramente monoica) y el sexo en los árboles usualmente es determinado por la presencia de conos (aunque también existen diferencias en la arquitectura de las ramas entre ambos sexos) (Grosfeld 1994). Los conos femeninos son producidos en la parte terminal de las ramas. Son muy grandes (entre 15 y $20 \mathrm{~cm}$ de diámetro) y contienen entre 100 y 200 semillas de $3.5 \mathrm{~g}$ de peso cada una (Muñoz Ibáñez 1984). Los conos son polinizados por el viento durante el verano y la maduración de las semillas demora de 16 a 18 meses. Esta fenología prolongada determina que entre el verano y el otoño puedan verse las dos generaciones consecutivas de conos. Las semillas se dispersan en otoño por gravedad a corta distancia y en primavera comienzan a germinar (Sanguinetti 2008). La semilla es un alimento valioso para la cotorra austral o cachaña (Enicognathus ferrugineus)(Müller) (Shepherd et al. 2008; Díaz et al. 2012). Sin embargo, el ensamble de varias especies de roedores nativos conforman el principal grupo de granívoros (Shepherd \& Ditgen 2005). Debido a la baja fertilidad, a la limitada dispersión de sus semillas y a la fuerte depredación que ejercen los granívoros, la especie presenta una capacidad de regeneración baja (Muñoz Ibáñez 1984; Sanguinetti \& Kitzberger 2009).

\section{Superficie de bosque y densidad de semilleros}

Dentro del área estudio de $1540 \mathrm{~km}^{2}$, la superficie de cada tipo de bosque de araucaria fue estimada por personal especializado del parque a partir del análisis de imágenes SPOT (10 m de resolución) provenientes de la CONAE (García, comunicación personal). La densidad de semilleros de araucaria se estimó a partir del método de los Cuadrantes Centrados, instalando un mínimo de 15 puntos de muestreo por población repartidos según los tipos de bosque (Aa_denso, Aa_Np, etc.) presentes, totalizando 150 puntos en las siete poblaciones donde se contaron los conos. En cada uno de los cuatro cuadrantes establecidos por punto a partir de líneas perpendiculares se identificaron las araucarias más próximas por cuadrante; se midió la distancia de estos individuos al punto de muestreo, su diámetro a la altura del pecho (Dap) y se identificó su condición reproductiva (femenino, masculino o inmaduro). Sólo se muestrearon los árboles con un Dap $\geq 20 \mathrm{~cm}$. A partir de 28 puntos de muestreo provistos por un trabajo previo
(Szymañski 2012) se pudo sumar tres poblaciones (Zapata, Cajón y Pulmarí) al conjunto total donde se estimó la producción de semillas por hectárea y ampliar la estimación de la densidad de semilleros en Calfiquitra (Tabla 1).

\section{Producción de conos y de semillas por árbol}

Cada verano entre los años 2000 y 2013 se obtuvieron datos sobre la producción de conos de árboles individuales a partir de la observación directa con binoculares y se seleccionaron árboles al azar a lo largo de sendas existentes en cada una de las siete poblaciones (como se describió en el trabajo previo) (Sanguinetti \& Kitzberger 2008). Los semilleros sin conos, "en reposo", fueron detectados a partir de las diferencias morfológicas en la disposición de las ramas entre ambos sexos y mediante la detección de estructuras reproductivas en años previos (Grosfeld 1994). Dada la coexistencia de ambas generaciones de conos en el mismo árbol y a que el aborto de conos en esta especie es un evento muy raro (Sanguinetti 2008), fue posible extender el registro hasta el 2014. Cuatro poblaciones fueron muestreadas durante todo el período, y desde 2002 y hasta 2004 se agregó anualmente una población al estudio. Por ende, se cuenta con un período común de 15 años para cuatro poblaciones y otro de 11 años (2004-2014) para las siete poblaciones. Cada año se muestrearon entre 50 y 100 semilleros por población. En promedio se censaron 670 árboles por año y un total de 9379 durante el periodo de estudio.

Para establecer el número de semillas por cono, durante el período 2000-2008 se descolgaron conos de los árboles utilizando tijeras largas o con la ayuda de los pobladores y el uso de lazos. Según la disponibilidad y accesibilidad de los conos, en los años que fue posible cosecharlos (en 2005 no fue posible) se muestrearon entre 19 y 31 conos totales de árboles diferentes por población. En las poblaciones TAQ y TRO por presentar árboles muy altos y de forma aparasolada no fue posible obtener conos.

\section{Análisis de los datos}

Para cada población se obtuvo el promedio anual del número de conos por árbol y los promedios globales por año para el período evaluado. A su vez, se estimó el promedio global por año del número de semillas por cono para el período 2000-2008, excepto en 2005, cuando no fue posible conseguir conos por su extraordinaria escasez.

A partir del análisis temporal de la producción de conos, y del cálculo de la media y de su EE, se clasificó la productividad de los años en "alta", "intermedia" y "baja". Se definieron como "Alta" y "Baja" a todo año con valores superiores o inferiores a la media del período más $1 \mathrm{EE}$, respectivamente. Los años con productividad "Intermedia" fueron definidos como aquellos con valores incluidos dentro del intervalo de la media $\pm 1 \mathrm{EE}$. 
La evaluación de diferencias en el número de conos por árbol entre población y su interacción con los años con distinto rango de productividad se analizó mediante Modelos Lineales Generalizados, GLMs) ajustando los datos a una distribución de Poisson y utilizando una función logarítmica como enlace. Por medio del software Statistica 7.0 se consideró al número de conos por árbol como variable dependiente y a la "Población" y a la "Clasificación de productividad anual" como factores. El efecto de factores de sitio sobre la variación espacio-temporal en la producción de conos por árbol, muestreados cada año al azar, también fue analizado mediante GLMs.

\section{Evaluación de factores que pueden influir la producción de conos a escala poblacional}

Se consideró como variable dependiente al número de conos por árbol en cada año para el período común 2004-2014 en los siete sitios, a la exposición (norte o sin exposición), a la composición del bosque (puro o mixto) y a la Clasificación de productividad anual (Baja, Intermedia o Alta) como factores y a la altitud o al área basal por hectárea (como indicador de la estructura) como covariables. Debido a la correlación significativa $(r=0.30 ; P<0.0001)$ entre el área basal y la altitud, ambas variables fueron ingresadas de manera separada en los análisis.

\section{Estimaciones de la producción de conos y semillas por hectárea y por población}

Para la estimación bruta de la producción de conos y semillas por hectárea y por población, se estimó la densidad de semilleros por población a partir del cálculo de la densidad total del bosque y de la proporción de semilleros en la muestra. A partir de la estimación de la densidad de semilleros en 38 lugares y del número de conos y de semillas por árbol en siete poblaciones, se estimó la producción de conos y semillas por unidad de superficie (ha). Utilizando el promedio del número de conos y semillas por hectárea para cada tipo de bosque (mixto o puro; denso o abierto) y con el apoyo del mapa detallado sobre la distribución de la especie, se estimó la producción de semillas para cada población (subcuenca) presente en el área de estudio a partir de la extrapolación de los datos a los parches donde no hubo muestreo ni de conos ni de densidad de semilleros.

La evaluación de las diferencias en el porcentaje de árboles en reposo o en el número de semillas por cono entre años así como el análisis estadístico de las diferencias en la producción bruta de semillas por hectárea y por población se realizó mediante Modelos Lineales Generales (ANOVA). Previo a los análisis los porcentajes fueron transformados aplicando el arcoseno de la raíz cuadrada mientras que al resto de las variables se les aplicó el logaritmo natural (ln valor+1) con el objeto de reducir la correlación entre la media y la varianza y para normalizar los datos.

\section{Resultados}

\section{Producción de conos y semillas por árbol y su variación temporal}

La producción promedio de conos contados por árbol para el período 2000-2014 fue de 19.4 $( \pm 1.3 \mathrm{EE})$, con una variación interanual de 56 veces (1 a 62 conos; Figura 2a). Los años de máxima producción fueron 2000, 2007 y 2013, mientras que los de mínima fueron 2005, 2008, 2012 y 2014 . El patrón reproductivo entre sitios fue altamente sincrónico (Figura 2b).

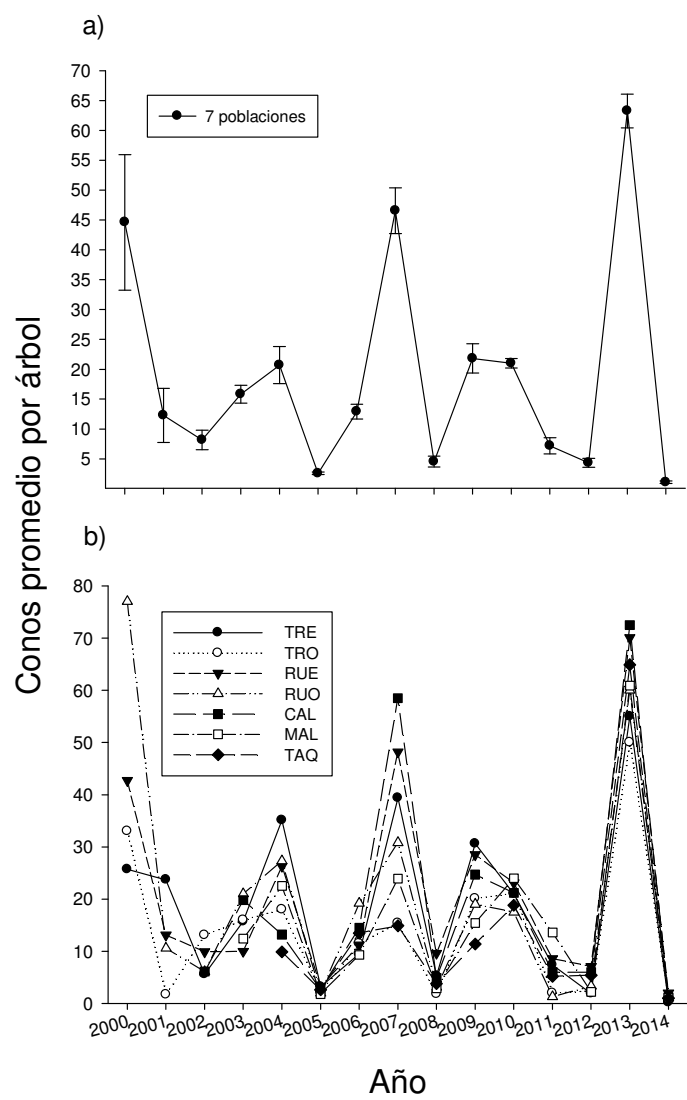

Figura 2. Producción media de conos $( \pm \mathrm{EE})$ : a) para el conjunto de datos en el período 2000-2014; b) para cada una de las siete poblaciones en dicho período.

Figure 2. Mean $( \pm S E)$ cone production: a) overall mean for the period 2000-2014; b) for each of the seven populations during 2000-2014.

El porcentaje promedio anual estimado de semilleros sin conos ("en reposo") fue $18.6 \%( \pm 2.2 \mathrm{EE}$; rango: $0-58 \%)$, y varió significativamente entre años $\left(F_{(13,94)}=45.6\right.$; $P<0.0001)$; en promedio, fue $36 \%$ en años de baja productividad y casi cero $(0.9 \%)$ en años muy productivos. La proporción de árboles "en reposo" en la población decrece de manera exponencial con el aumento del número de conos por árbol, y asume valores cercanos a 
cero a partir del promedio de 42 conos por árbol.

La producción promedioestimada desemillas por árbol fue 2372 ( \pm 701 EE), con una variación interanual de 152 veces (61-9287 semillas). La mayor variación temporal en la producción de semillas respecto a la de conos se debió al hecho de que hubo significativamente más semillas por cono en los años más productivos $\left(F_{(7,260)}=15.2 ; P<0.0001\right)$. Por ejemplo, en 2000 hubo 135 $\pm 8(\mathrm{EE} ; n=31)$ semillas / cono; en 2001, $90 \pm 12(\mathrm{EE}, n=19)$; en 2002, $120 \pm 5$ (EE; $n=53)$, y en 2008, 58 \pm 9 (EE; $n=22)$.

En años de "Baja", "Intermedia" y "Alta" productividad en los árboles se contó en promedio 5.2 ( $\pm 0.7 \mathrm{EE}$; rango: 3.9-8.6), $17( \pm 1$; rango: $12.4-20)$ y 42.9 ( $\pm 2.6 \mathrm{EE}$; rango: 33.9$47.8)$ conos, respectivamente, y produjeron una estimación media de 462 ( $\pm 58 \mathrm{EE}$; rango: 367-704), 1844 ( $\pm 82 \mathrm{EE}$; rango: 1520-2088) y 6050 ( \pm 359 EE; rango: 4740-6956) semillas / árbol, respectivamente.

En el período común 2004-2014 se detectaron diferencias significativas entre sitios (Wald $\left.\chi_{(60)}^{2}=2122 ; P<0.0001\right)$. En los sitios CAL y RUE se encontraron, en promedio, más conos/árbol que en el resto de los sitios $(P<0.0001)$, mientras que TAQ presentó los árboles con menos conos en promedio, aunque sólo significativamente distinto a CAL, RUE y RUO (Figura 3a). El patrón de disminución en la productividad de conos es coincidente con el eje longitudinal (EsteOeste; Figura 3a). Se detectaron variaciones significativas entre sitios y años según el nivel de productividad (Wald $\chi_{(12)}^{2}=3855$; $P<0.0001)$. En años de "Alta" productividad, en CAL se contaron significativamente más conos que en el resto de los sitios $(P<0.001)$. En años con productividad "Intermedia", en TRE se contaron más conos que en casi todos los sitios (excepto RUE; $P<0.001$ ), y en TAQ se contaron significativamente menos conos que en los otros lugares $(P<0.001)$. Mientras tanto, en años de "Baja", en RUE-CAL se contaron más conos que en TRO-TRE-MAL $(P<0.001)$, y en TAQ, más conos que en TROTRE $(P<0.01)$.

\section{Evaluación de factores que pueden influir la producción de conos a escala poblacional}

De las dos variables continuas evaluadas, sólo el área basal/ha presentó un efecto significativo sobre la producción de conos al observarse una tendencia negativa en los tres
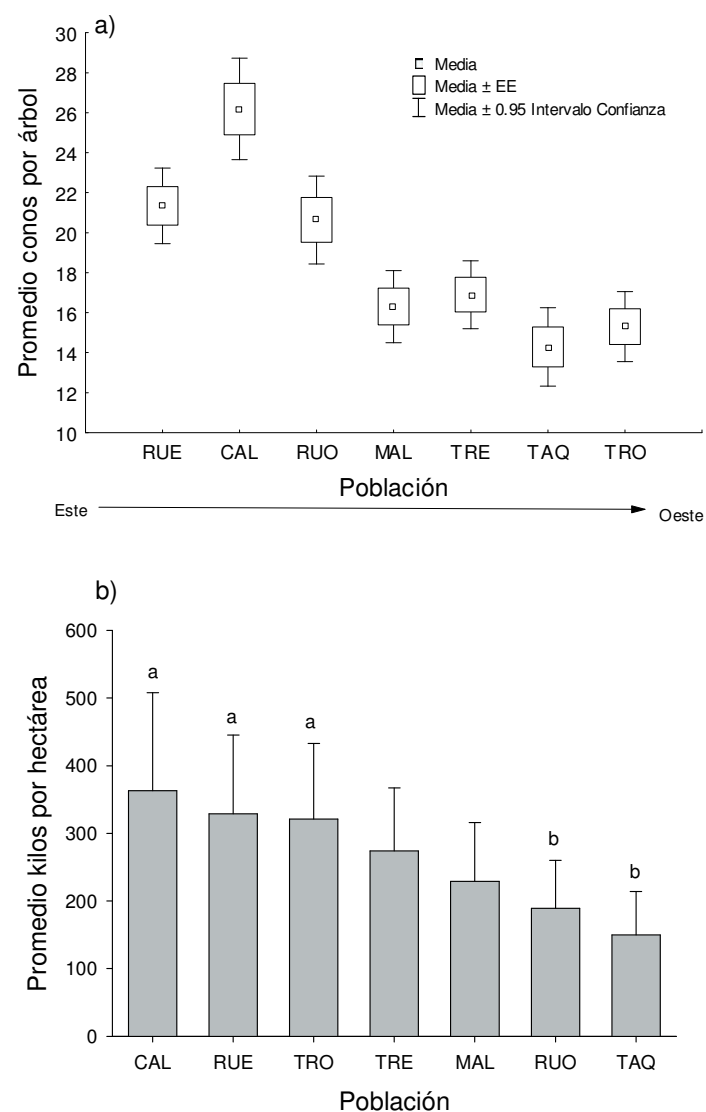

Figura 3. Variación entre poblaciones en la producción de semillas por árbol y por hectárea para el período común 2004-2014: a) logaritmo natural del número promedio $( \pm E E)$ de conos +1 por árbol, $\mathrm{y} ; \mathrm{b}) \mathrm{kg}$ de semillas /ha (promedio $\pm \mathrm{EE}$ ). Las letras diferentes indican diferencias significativas $(P<0.001)$. En a) los sitios están ordenados de este a oeste.

Figure 3. Population variation on seed production per tree and per hectare for the common period 2000-2014: a) natural logarithm of mean $( \pm \mathrm{SE})$ cones +1 per tree, and; $b)$ mean $( \pm$ SE) $\mathrm{kg}$ seeds $/ \mathrm{ha}$. Significant differences $(P<0.001)$ are shown by different letters. On a) sites are ordered from East to West.

tipos de años productivos definidos (Tabla 2). Sin embargo, el ajuste predictivo de esta variable es muy pobre (en las tres clases de productividad siempre el $r^{2}$ fue $<0.03$ ). La altitud mostró una tendencia positiva y negativa respecto a la producción de conos en años de productividad "Alta" e "Intermedia", respectivamente, y sin tendencia en años de "Baja".

El número de conos contados por árbol varió significativamente en sitios con distinta exposición solar y en menor medida en bosques con diferente composición forestal (Tabla 2). Se contaron más conos en árboles ubicados en exposición norte (Figura 4a) y marginalmente más en bosques mixtos que en los puros. Sin embargo, los efectos de la 

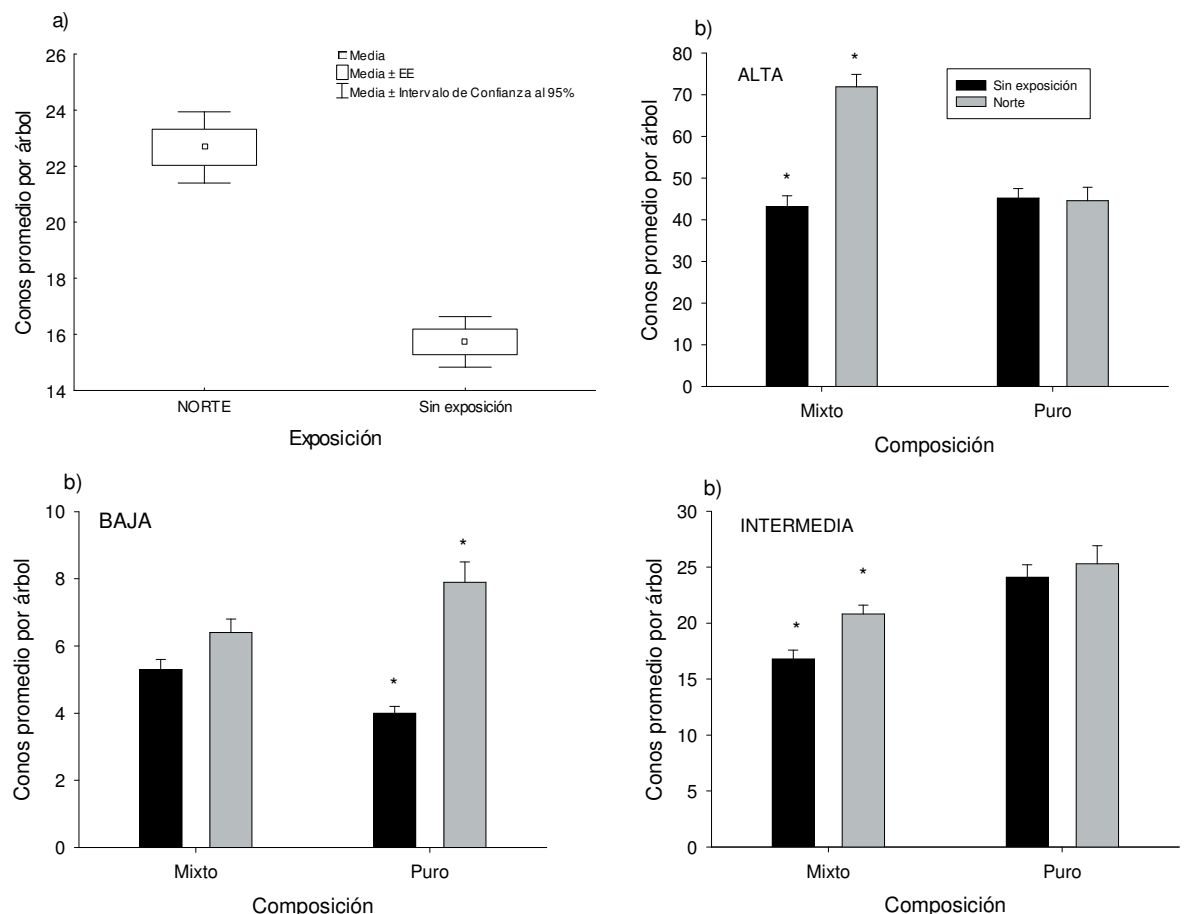

Figura 4. Efectos de la exposición, y su interacción con la composición forestal sobre el número de conos/árbol en años con distinto nivel de productividad: a) promedio ( \pm EE) e Intervalo de confianza para el número de conos por árbol en individuos creciendo en exposición norte y sin exposición; b) número promedio ( $\pm \mathrm{EE}$ ) de conos/árbol según exposición y composición forestal y en años con alta, intermedia y baja productividad en la producción de conos. " $P<0.001$

Figure 4. Aspect, and their interaction with forest composition, on cone number/tree during years with different cone production productivity: a) mean $( \pm \mathrm{SE})$ and confident intervals for cone number/tree on individuals growing on different aspect; $b)$ mean $( \pm S E)$ cones per tree according to aspect and forest composition and during high, regular and poor cone productive years. ${ }^{*} P<0.001$

exposición y de la composición forestal no fueron homogéneos en las tres clases de años de productividad (Tabla 2; Figura 4b). En años de productividad "Alta" e "Intermedia", el efecto de la exposición sólo se observó en

Tabla 2. Efectos de la altitud o Área basal/ha (covariables), de la exposición, composición y clase de años según productividad sobre la producción de conos por árbol examinado mediante Modelos Lineales Generalizados (GLMs) con datos obtenidos entre 2004-2014 en siete poblaciones de araucaria.

Table 2. Altitude or basal area/ha (covariables), aspect, forest composition and cone productive year class effects on cone production per tree evaluated by Generalized Lineal Model (GLMs) with data obtained during 20042014 period on seven araucaria populations.

\begin{tabular}{lccccc}
\hline & \multicolumn{3}{c}{ c/Altitud } & \multicolumn{2}{c}{ c/Área Basal } \\
& gl Wald ? & $\mathrm{P}$ & Wald ? & $\mathrm{P}$ \\
\hline Altitud o Área & 1 & 3.5 & 0.06 & 310 & $<0.0001$ \\
Basal & & & & & \\
Clase de año & 1 & 67799 & $<0.0001$ & 67666 & $<0.0001$ \\
Exposición & 2 & 1438 & $<0.0001$ & 1246 & $<0.0001$ \\
Composición & 1 & 0.03 & 0.87 & 5.5 & $<0.05$ \\
Año*Exposición & 2 & 297 & $<0.0001$ & 283 & $<0.0001$ \\
Año*Composición $^{*}$ & 2 & 1397 & $<0.0001$ & 1396 & $<0.0001$ \\
Exposición* $^{*}$ & 1 & 19.7 & $<0.0001$ & 96 & $<0.0001$ \\
Composición & & & & & \\
\hline
\end{tabular}

árboles ubicados en bosques mixtos, mientras que en años de "Baja" lo fue sólo en bosques puros (Figura $4 b$ ).

\section{Producción bruta de conos y semillas por hectárea y por población}

En el período 2000-2014, la producción promedio por hectárea, estimada en las siete poblaciones, fue 685 conos $( \pm 166 \mathrm{EE}$; rango: $38-2223)$ y 89394 semillas $( \pm 18903 \mathrm{EE}$; rango: 2958-415730) (Tabla 3). El promedio estimado de conos/ha en años de producción Baja, Intermedia y Alta fueron 211 ( $\pm 53 \mathrm{EE}), 653$ ( $\pm 81 \mathrm{EE})$ y $1546( \pm 296 \mathrm{EE})$, respectivamente. La producción promedio estimada de $\mathrm{kg} / \mathrm{ha}$ en 15 años fue 273 ( \pm 75 EE; rango: 13-1052).

En el período común 2004-2014, la estimación de producción promedio de kilos de semillas/ha varió significativamente entre sitios $\left(F_{(6,60)}=8.16 ; P<0.0001\right.$; Figura $3 b)$. Los sitios CAL, RUE y TRO tuvieron una productividad promedio de conos o semillas/ha significativamente mayor que TAQ y RUO. 
Tabla 3. Número de conos y semillas promedio ( \pm EE) por árbol en las poblaciones evaluadas entre 2000 y 2014.

Table 3. Mean $( \pm$ SE) cones and seeds per tree for seven populations sampled during the period 2000-2014.

\begin{tabular}{cccccc}
\hline Población & Conos/árbol & Semillas/árbol & Conos/ha & Semillas/ha & Período \\
\hline TRE & $19.4( \pm 4.7)$ & $2369( \pm 667)$ & $680( \pm 159)$ & $82906( \pm 23347)$ & $2000-2014$ \\
TRO & $15.7( \pm 3.9)$ & $1952( \pm 567)$ & $817( \pm 194)$ & $101503( \pm 29487)$ & $2000-2014$ \\
RUE & $21.2( \pm 5.3)$ & $2619( \pm 796)$ & $805( \pm 194)$ & $99541( \pm 30231)$ & $2000-2014$ \\
RUO & $21.1( \pm 6.2)$ & $2657( \pm 870)$ & $549( \pm 56)$ & $69093( \pm 22613)$ & $2000-2014$ \\
CAL & $24.1( \pm 7.8)$ & $3031( \pm 1210)$ & $851( \pm 250)$ & $107047( \pm 38726)$ & $2002-2014$ \\
MAL & $16.5( \pm 5.3)$ & $2080( \pm 839)$ & $567( \pm 152)$ & $70387( \pm 24063)$ & $2003-2014$ \\
TAQ & $16.1( \pm 5.9)$ & $2077( \pm 938)$ & $370( \pm 116)$ & $47068( \pm 18472)$ & $2004-2014$ \\
Promedio & $19.4( \pm 1.3)$ & $2399( \pm 134)$ & $685( \pm 166)$ & $85232( \pm 25590)$ & \\
\hline
\end{tabular}

La producción bruta total de semillas por población varió significativamente como consecuencia de diferencias en la productividad por unidad de superficie y por variaciones entre sitios en la extensión de bosque de araucaria $\left(F_{(6,70)}=3.35 ; P<0.01\right.$; Tabla 4). En el período común 2004-2014, para las siete poblaciones se estimó un promedio de $65 \mathrm{t}$ de semillas ( $\pm 24 \mathrm{EE}$; rango: 8-188) en una superficie promedio neta de araucaria de 255 ha (rango: 35-482). La producción bruta estimada por extrapolación en las restantes tres poblaciones varió entre 29 y 187 t (Tabla 4).

\section{Variación de la producción de semillas entre tipos de bosque}

La producción promedio estimada de $\mathrm{kg}$ semilla/ha varió significativamente entre los distintos tipos de bosque estudiados $\left(F_{(5,27)}=3.34 ; P=0.018\right.$; Figura 5). Los bosques densos de araucaria (Aa denso) y los de araucaria-lenga (Aa_Np) son, al menos, 2.5 y 1.5 veces más productivos que los restantes tipos forestales, respectivamente $(P<0.01$; Figura 5). No se encontraron diferencias significativas entre los bosques mixtos de araucaria y ñire y aquellos donde la conífera

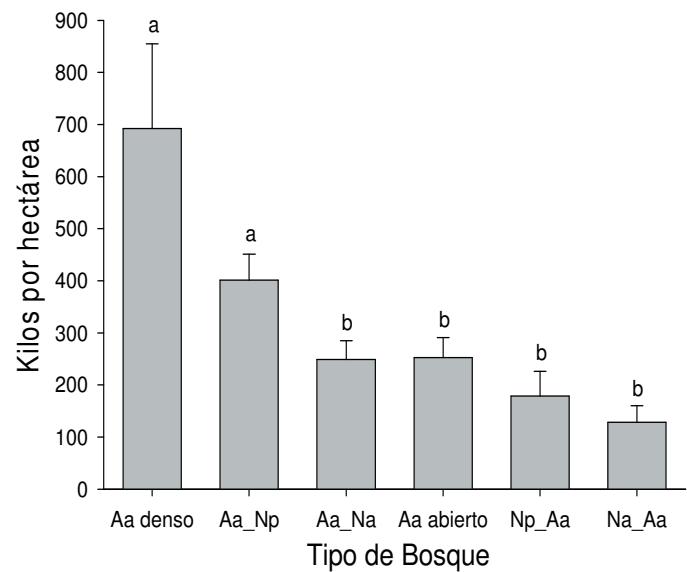

Figura 5. Peso promedio de semillas de araucaria/ha $( \pm \mathrm{EE})$ en bosques monoespecíficos o mixtos de AraucariaNothofagus spp. dominados o no por la conífera. Araucaria denso (Aa_denso), Araucaria-N. pumilio (Aa_Np), Araucaria-N. antarctica (Aa_Na), Araucaria abierto (Aa_ abierto), N. pumilio-Araucaria (Np_Aa) y N. antarticaAraucaria (Na_Aa). Letras diferentes indican diferencias significativas $(P<0.05)$

Figure 5. Mean $( \pm \mathrm{SE})$ araucaria seed $\mathrm{kg} / \mathrm{ha}$ on pure or mixed Araucaria-Nothofagus spp. forests where the conifer dominates or not. Dense Araucaria (Aa_denso), AraucariaN.pumilio (Aa_Np), Araucaria-N. antarctica (Aa_Na), Open Araucaria (Aa_abierto), N. pumilio-Araucaria (Np_Aa) and N. antarctica-Araucaria (Na_Aa). Significant differences $(P<0.05)$ are shown by different letters.

Tabla 4. Producción promedio de toneladas totales por población durante años "Baja", "Intermedia" y "Alta" en el período 2000-2014. Los valores de PUL, ZAP y CAJ fueron calculados a partir de la extrapolación de los datos promedio sobre conteo de conos y semillas en las restantes poblaciones.

Table 4. Seed gross production (tonnes) for the sampled populations during "Poor", "Regular" and "Productive" years during 2000-2014. The values for PUL, ZAP and CAJ were estimated using cones per tree and seeds per cone mean values obtained from the other populations.

\begin{tabular}{ccccccc}
\hline Población & $\begin{array}{c}\text { Superficie } \\
\text { (ha) }\end{array}$ & $\begin{array}{c}\text { Semilleros } \\
(\text { árboles } / \text { ha) }\end{array}$ & Baja & $\begin{array}{c}\text { Toneladas totales } \\
\text { Intermedia }\end{array}$ & Alta & $\begin{array}{c}\text { Promedio } \\
2004-2014\end{array}$ \\
\hline TRE & 48 & 35 & 4 & 11 & 29 & $13( \pm 3)$ \\
TRO & 61 & 52 & 6 & 19 & 44 & $20( \pm 5)$ \\
RUE & 206 & 38 & 23 & 50 & 157 & $68( \pm 24)$ \\
RUO & 481 & 26 & 20 & 84 & 275 & $91( \pm 34)$ \\
CAL & 517 & 44 & 38 & 160 & 475 & $188( \pm 75)$ \\
TAQ & 432 & 23 & 12 & 50 & 167 & $65( \pm 28)$ \\
MAL & 35 & 35 & 2 & 6 & 19 & $8( \pm 3)$ \\
ZAP & 353 & 70 & 48 & 181 & 579 & $187( \pm 52)$ \\
PUL & 453 & 41 & 36 & 135 & 432 & $140( \pm 38)$ \\
CAJ & 50 & 78 & 8 & 28 & 91 & $29( \pm 8)$ \\
Promedio & $271( \pm 62)$ & $43( \pm 6)$ & $20( \pm 5)$ & $73( \pm 20)$ & $227( \pm 64)$ & $81( \pm 29)$ \\
\hline
\end{tabular}


es acompañante. Las variaciones en la productividad entre bosques se explicó por la diferencia significativa en la densidad de semilleros $\left(F_{(5,36)}=5.35 ; P<0.001\right)$ y no por diferencias en el tamaño (Dap) de los mismos $\left(F_{(5,36)}=2.18 ; P=0.08\right)$.

\section{DisCUSIÓN}

En el presente trabajo se muestra que el patrón espacio-temporal de la producción de conos en araucaria está influenciado primariamente por la exposición solar y de manera secundaria por la composición y la estructura forestal. Los árboles más productivos están ubicados en poblaciones con exposición norte y en altitudes intermedias y en bosques mixtos con valores moderados de área basal/ha. Araucaria, al parecer, presenta picos máximos de producción de conos cada 6-7 años y una variación media de 8 veces entre años de Alta y Baja productividad.

Existiría un patrón espacial caracterizado por la reducción de la producción de conos por árbol de este a oeste, probablemente en relación con el incremento de la altitud, del área basal/ha y de la pérdida del efecto de la exposición solar con el incremento de la densidad del bosque hacia el oeste. Sanguinetti \& Kitzberger (2008) determinaron que la sequía 2 años antes de la producción de conos sería la clave ambiental que dispara la diferenciación de yemas para la formación de conos en forma sincrónica entre árboles y entre poblaciones. Las diferencias en el efecto de la exposición norte (seca) entre años de "Alta", "Intermedia" y "Baja" productividad en bosques mixtos respecto a los puros se explicarían por la ubicación de estos bosques respecto al gradiente de humedad y al nivel de estrés hídrico que éstos perciben. Por lo general, los bosques mixtos se ubican a mayor altitud y hacia el oeste; por ende, los árboles en estos bosques "sentirán" con mayor intensidad el estrés hídrico que estimularía la formación de yemas florales sólo en años de sequía extrema y en la exposición más seca de la montaña. Por el contrario, los años de "Baja" productividad están asociados con una humedad fuerte al momento de la inducción floral, y sólo los bosques ubicados en sitios más secos y a menor altitud, como los bosques puros en exposición norte, recibirían el estímulo climático. A su vez, los bosques puros mostrarían mayores niveles de competencia intraespecífica y/o limitaciones de nutrientes al presentar una mayor densidad de individuos y crecer sobre suelos usualmente rocosos; esto explicaría la falta de respuesta de los semilleros al efecto "exposición" en años de productividad "Alta" e "Intermedia".

Si bien entre poblaciones hubo diferencias en el número de conos/árbol durante los años de mínima y máxima productividad, la variación en la producción entre poblaciones se debió no tanto a diferencias en la escala de árbol, sino a las diferencias por hectárea y por población debido a la variabilidad en la densidad de semilleros y en la superficie neta de araucaria. Las poblaciones con mayor productividad promedio por unidad de superficie (e.g., CAL, RUE o TRO) son aquellos que tienen árboles más productivos o una mayor densidad de semilleros. Los bosques densos (>200 árboles / ha) y puros de araucaria compensarían la existencia de árboles menos productivos con la mayor densidad de semilleros.

Las diferencias de productividad entre árboles ubicados en distintas poblaciones podrían estar relacionadas con el tamaño, la forma y el volumen de copa de los árboles y con la competencia con los árboles vecinos más cercanos. A futuro se considera importante avanzar en esta línea de investigación para dilucidar aún más la importancia de estos factores intrínsecos o locales.

Las estimaciones en este trabajo del número promedio de semillas / ha en el período 20042010 serían 2 veces más altas que el promedio publicado en Chile para los mismos años (119600 vs 51835 semillas/ha) (Donoso et al. 2010). A su vez, la productividad estimada es siempre superior a las publicadas en Chile en décadas previas (Muñoz 1984; Caro Caro 1995). Esto podría indicar que los bosques evaluados en el Parque Nacional Lanín en Argentina serían más productivos que los estudiados en Chile en la comuna de Lonquimay. De estos estudios también se desprende que existiría no sólo una sincronización regional de todas las poblaciones en Argentina (Sanguinetti \& Kitzberger 2008), sino que éstas también estarían sincronizadas con las poblaciones chilenas.

La mayor variabilidad en la producción de conos a escalas espaciales crecientes podría tener implicancias importantes sobre el patrón de ocurrencia y abundancia de la regeneración en esta especie, que se distribuye en forma fragmentada por factores ecológicos intrínsecos y por perturbaciones humanas. A pesar del extraordinario nivel de sincronía reproductiva, es posible que los parches más pequeños de araucaria tengan, 
proporcionalmente por unidad de superficie, mayores presiones de granivoría y, por lo tanto, menores niveles de regeneración. Por esto se esperaría que sólo en los bosques más extensos exista "escape espacial" frente a la granivoría producida por animales móviles. Esto fue verificado en bosques muy densos y productivos como TRO, donde la fluctuación en la producción de conos estaría saciando a especies invasoras como Rattus norvergicus (Berkenhout) (Shepherd \& Ditgen 2005) y Sus scrofa (Linnaeus) (Sanguinetti \& Kitzberger 2010). Por el contrario, en bosques menos productivos y con mayor presión de granivoría por ganado y por el hombre, la supervivencia de semillas es mínima o nula (Sanguinetti 2008).

En las últimas décadas, la competencia por la semilla de araucaria con la fauna doméstica y las demandas del mercado por el recurso han alentado al humano a realizar la cosecha desde el árbol utilizando diversas técnicas para golpear los conos con lazos, gomeras o varas. Estas técnicas aumentan significativamente la eficacia de la cosecha y permiten la colecta del 50-75\% de conos/árbol (Hermann 2006). En 1200 ha netas de araucaria en Rucachoroy, la cuenca más utilizada del norte del Parque Nacional Lanín, el ser humano anualmente cosecha entre 10 y 80 t de semillas. Según los resultados de este trabajo, estos valores representarían entre 5 y 25\% de la producción bruta, siendo mayor la presión en los años de baja productividad. En esta zona se observa con claridad una tendencia alcista de los volúmenes de cosecha desde 1993 a 2013, vinculados con el aumento de la población humana y con el cambio en las técnicas de cosecha (datos estadísticos del Parque Nacional Lanín).

En el marco de planes de manejo para las áreas utilizadas por los Mapuches, tanto dentro del Parque Nacional Lanín como en el resto de la provincia del Neuquén, la información generada en este trabajo puede servir para fijar cuotas de cosecha de semillas según la productividad en cada sector para garantizar que sobre el recurso para la fauna y para la regeneración. El preocupante aumento del ganado en los bosques y de especies silvestres invasoras consumidoras de la semilla (i.e., jabalí, ciervo colorado, liebre, conejo y rata noruega) amerita con urgencia que el Estado arbitre los medios para minimizar estas amenazas que ponen en duda la conservación de largo plazo de este increíble, frágil y raro ecosistema.
Agradecimientos: Se agradece el apoyo en el trabajo de campo a los guardaparques C. Reta, F. Nahuelpan, J. Delgado, A. Dáros, P. Sugliano, D. Schro, N. Ferrari y J. Lenz; a los estudiantes A. Androsiuk, C. Syzmañski, G. Fontana y a los voluntarios aportados por Global Vision International (GVI). Al apoyo en gabinete de L. García y G. Miño. Este trabajo fue financiado parcialmente por Russell Train Scholarship (WWF), por el gobierno Alemán (GMZ), por el Instituto Internacional sobre Recursos Genéticos en Plantas (IPGRI) y por la Agencia de Cooperación Española (AECI) y por el Parque Nacional Lanín.

\section{BibLIOGRAFÍA}

Caro Caro, MP. 1995. Producción y dispersión de semillas de Araucaria araucana en Lonquimay. Memoria para optar al título profesional de ingeniero Forestal. Escuela de Ciencias Forestales. Facultad de Ciencias Agrarias y Forestales. Universidad de Chile.

DE FINA, AL. 1972. El clima de la región de los bosques andinopatagónicos argentinos. La región de los bosques andinopatagónicos. Dimitri, MJ (ed.). INTA, Buenos Aires

Díaz, S; T Kitzberger \& S Peris. 2012. Food resources and reproductive output of the Austral Parakeet (Enicognathus ferrugineus) in forests of northern Patagonia. Emu, 112:234-243.

Donoso, S; K Peña-Rojas; C Pacheco; F Perry \& C Espinoza. 2010. Evolución de la sustentabilidad de los bosques de Araucaria araucana: Producción, colecta y consumo de piñones. Spanish Journal of Rural Development, 1:99-112.

FIA. 2010. Resultados y lecciones en productos a partir del piñon. Proyecto de Innovación en Regiones del Biobío y de La Araucanía. Serie Experiencias de Innovación para el Emprendimiento Agrario $\mathrm{n}^{\circ}$ 76. Fundación para la Innovación Agraria. Ministerio de Agricultura, Chile. Pp. 48.

FINCKH MA \& A PAULSCH. 1995. The ecological strategy of Araucaria araucana. Flora, 190:365-382.

GROSFELD, JE. 1994. Arquitectura y dinámica del crecimiento de Araucaria. Tesis de Licenciatura en Ciencias Biológicas, Universidad Nacional del Comahue.

HARPER, JL. 1977. Population biology of plants. Academic press, London, U.K.

Herrera, CM. 1998. Population-level estimates of interannual variability in seed production: what do they actually tell us? Oikos, 82:612-616.

Herrmann, TM. 2006. Indigenous knowledge and management of Araucaria araucana forest in the Chilean Andes: implications for native forest conservation. Biodivers. Conserv., 15:647-662.

Lara, A; C Donoso \& IC ARavena. 1995. La conservación de los bosques nativos de Chile: problemas y desafíos, Capítulo 8: 335-362. En: ARMesto, J; C Villagran \& MK Arroyo (eds.). Ecología de los bosques nativos de Chile. Comité de Publicaciones Científicas, Vicerrectoría Académica, Universidad de Chile. Pp. 477.

MatTHEWs, JD. 1963. Factors affecting the production of seed by forest trees. For. Abstr., 24:1-13.

Muñoz Ibañez, R. 1984. Análisis de la productividad de semillas de Araucaria araucana (Mol.) C. Koch, en el Area de Lonquimay - IX Región. Tesis para optar al Título de Ingeniero Forestal. Facultad de Ciencias Agrarias, Veterinarias y Forestales. Escuela de Ciencias Forestales, Universidad de Chile.

OWENS, JN \& MD BLAKE. 1985. Forest tree seed production. can. for. serv. petawawa nat. for. inst. chalk river, ontario, inf. rep. Pi-x-53. Pp. 161. 
Rechene, C; J Bava \& R Mujica. 2003. Los bosques de Araucaria araucana en Chile y Argentina. Programa de Apoyo Ecológico (TOEB), GTZ, Agencia de Cooperación Alemana, Informe TWF-V /40s, Eschborn, Alemania.

SANGUinetTI, J. 2008. Producción y predación de semillas, efectos de corto y largo plazo sobre el reclutamiento de plántulas. Caso de Estudio: Araucaria araucana. Tesis para optar al título de Doctor en biología, Universidad Nacional del Comahue, Bariloche.

Sanguinetti, J \& T Kitzberger. 2008. Patterns and mechanisms of masting in the large-seed southern conifer Araucaria araucana. Austral Ecol., 33:78-87.

Sanguinetti, J \& T Kitzberger. 2009. Efectos de la producción de semillas y la heterogeneidad vegetal sobre la supervivencia de semillas y el patrón espaciotemporal de establecimiento de plántulas en Araucaria araucana. Rev. Chil. Hist. Nat., 82:319-335.

SAnguinetti, J \& T Kitzberger. 2010. Factors controlling seed predation by rodents and non-native Sus scrofa in Araucaria araucana forests: potential effects on seedling establishment. Biol. Invasion, 12:689-706.

SHEPHERD, JD \& RS Ditgen. 2005. Human Use and Small Mammals Communities of Araucaria Forests in Neuquén Argentina. Mastozoología Neotrop., 12:217:226.

Shepherd, JD; RS Ditgen \& J SAnguinetti. 2008. Araucaria araucana and the Austral Parakeet: pre-dispersal seed predation on a masting species. Rev. Chil. Hist. Nat., 81:395-401.

SZYMAÑSKI, C. 2012. Evaluación ambiental y propuesta de manejo para el uso leñero y ganadero en un área de amortiguación del Parque Nacional Lanín en tierras de la Comunidad Mapuce Aigo. Tesina para optar al título de Ingeniera en Recursos Naturales Renovables. Facultad de Ciencias Agrarias, Universidad Nacional de Cuyo (Mendoza-Argentina). Pp. 97.

Veblen, TT. 1982. Regeneration patterns in Araucaria araucana forest in Chile. J. Biogeogr., 9:11-28.

Veblen, TT; RB Burns; T Kitzberger; A Lara \& R Villalba. 1995. The ecology of the conifers of southern South America. En: ENRIGHT N \& R Hill (eds.). Ecology of the Southern Conifers. Melbourne University Press, Parkville, Australia.

WORRell, R \& DC MALCOLM. 1989. Productivity of sitka spruce in northern britain. 1. The effect of elevation and climate. Forestry, 63:105-118.

Zamorano-Elgueta, C; L Cayuela; M GonZÁlez-Espinoza; A LARA \& MR PARRA-VAZQUEZ. 2012. Impacts of cattle on the South American temperate forests: challenges for the conservation of the endangered monkey-puzzle tree (Araucaria araucana). Biol. Conserv., 152:110-118. 